\title{
Human Immunodeficiency Virus (HIV)- Blood Interactions in Antiretroviral Drugs Environment-Surface Thermodynamics Approach
}

\author{
Okwuchukwu Innocent Ani ${ }^{1}$, Sam Nna Omenyi ${ }^{2}$, Chinonso Hubert Achebe ${ }^{2}$ \\ ${ }^{1}$ Department of Mechanical and Production Engineering, Enugu State University of Science and Technology, \\ Enugu, Nigeria \\ ${ }^{2}$ Department of Mechanical Engineering, Nnamdi Azikiwe University, Awka, Nigeria \\ Email: okwyenoch@yahoo.com,sam.omenyi@unizik.edu.ng,chinobert2k@yahoo.com
}

Received 12 September 2015; accepted 2 November 2015; published 5 November 2015

Copyright $@ 2015$ by authors and Scientific Research Publishing Inc.

This work is licensed under the Creative Commons Attribution International License (CC BY). http://creativecommons.org/licenses/by/4.0/

(c) (i) Open Access

\section{Abstract}

Hamaker coefficient approach was used as a surface thermodynamic tool in determining the HIVblood interactions in the antiretroviral drug environment. The methodology involved the absorbance measurement using a digital Ultraviolet Visible MetaSpecAE1405031Pro Spectrophotometer of blood samples collected from ten HIV infected persons who had not commenced treatment with antiretroviral drugs (No ARV), ten HIV infected persons who had already commenced treatment with antiretroviral drugs (with ARV) and ten blood samples of uninfected persons all in each of five different antiretroviral drugs environment. The variables required for the computations with the Lifshiftz formula in the determination of Hamaker constants/coefficients were derived from the absorbance data. The values of the various Hamaker coefficients for each antiretroviral drug on both infected and uninfected blood samples were calculated. MATLAB software tools were employed in the computations. The absolute values for the combined Hamaker coefficient for the drugs ranged from $-0.02481 \times 10^{-21}$ Joule for drug 4 to $-0.05845 \times 10^{-21}$ Joule for drug 3 . The negative senses of the absolute combined Hamaker coefficient imply net negative van der Waals forces indicating a possible repulsion between HIV and drug coated lymphocyte cells. For the virus interacting with blood samples not coated with the drugs, the Hamaker coefficients are positive indicating the vulnerability of the lymphocytes in the absence of the drugs. This effect suggests effective coating or binding of the lymphocytes with the drugs is needed for possible blocking of the virus from the surface of the lymphocyte cell. A thermodynamic criterion for HIV-drug interaction prediction was suggested and found to be a valuable tool in HIV-blood interaction study. The use of the findings of this work by pharmaceutical industries may be possible in the area of drug design. 
Keywords

\section{Absorbance, Hamaker Constant, Hamaker Coefficient, Human Immunodeficiency Virus, Antiretroviral Drug, Lymphocyte, Van der Waals Forces, Surface Energy}

\section{Introduction}

The UNAIDS Global report on HIV/AIDS treatment in 2013 [1] declared that an estimated 35.5 (32.2 - 38.8) million people were living with HIV in 2012. About 15 million people with HIV were expected to be reached with the lifesaving antiretroviral treatment by 2015 . Hence, the world is within the reach of providing antiretroviral therapy to a good number of HIV infected people across the globe. In 2012, 9.7 million people in low and middle income countries received antiretroviral therapy. Antiretroviral therapy not only prevents AIDS-related illness and death: it also has the potential to significantly reduce the risk of HIV transmission and the spread of tuberculosis. From 1996 to 2012, antiretroviral therapy averted 6.6 million AIDS-related deaths worldwide, including 5.5 million deaths in low and middle income countries. Antiretroviral therapy reduces the risk that a person living with HIV will develop tuberculosis [1].

It could also be noted that the antiretroviral regimens have yet to completely and permanently suppress the virus in HIV-infected people. Although, many antiretroviral drugs are being manufactured for the eradication of the HIV infections; but approximately 40,000 new HIV infections occur each year in the United States according to the Joint United Nations Programmes [2]. The power of highly active antiretroviral therapy (HAART) to suppress HIV has revolutionized the clinical management of HIV disease in the developed world [3]. The capacity for HIV to develop resistance to antiretroviral drugs, however, is a significant cause of the apparent failure of HAART. Genotypic and phenotypic resistance tests have the potential to help identify which drugs in a regimen are failing and to guide the selection of drugs for new regimens [4].

There are several classes of drugs, which are usually used in combination, to treat HIV infection. Use of these drugs in combination is termed anti-retroviral therapy (ART), combination anti-retroviral therapy (cART) or highly active anti-retroviral therapy (HAART). Anti-retroviral (ARV) drugs are broadly classified by the phase of the retrovirus life-cycle that the drug inhibits. Typical combinations include 2 NRTIs as a "backbone" along with 1 NNRTI or PI as a "base" [5] [6].

The increasing rate of HIV infection globally is blamed on the apparent ineffectiveness of some available antiretroviral therapy to block or resist perfectly this virus from invading the white blood cells (lymphocytes). The mechanism by which drugs can block the virus is well known but the drugs do not appear to summarily block the virus and hence it becomes necessary to study the interaction between the HIV and white blood cells in the presence of administered drugs from a different point of view-surface thermodynamics.

We intend to use absorbance data to calculate thermodynamic parameters in HIV-blood interactions. Achebe and Omenyi [7] have shown that absorbance is a surface phenomenon. They showed that the peak absorbance of the surface of each blood component was reduced by the presence of the virus. The question now arises as to what extent the peak absorbance of the surface of a given HIV infected blood component is changed by the administration of the anti retroviral drugs and what that means in terms of HIV-blood interactions?

HIV-blood interactions can be understood in terms of the surface energies (in energy units per unit area) of the interacting systems which quantify the disruption of intermolecular bonds that occur when a surface is created, that is, the energy associated with the intermolecular forces at the interface between two media. The term surface energy is used because an increase in the surface area of a solid cannot be accomplished without doing work against the elastic forces and plastic resistance of the solid [8].

The presence of an interface influences generally all thermodynamic parameters of a system. There are two models that are commonly used to demonstrate interfacial phenomena, which include the Gibbs ideal interface model and the Guggenheim model. Thermodynamically, the surface energy, $\gamma$ is interpreted as the increase in the Gibbs energy of the system when the area of the interface under consideration is increased reversibly by an infinitesimal amount $d A$ at constant temperature $(T)$, pressure $(\mathrm{P})$ and composition (c) [9]. This can be expressed as $\gamma=\left(\frac{\delta G}{\delta A}\right)_{T, p, c}$. In other words, surface energy can be interpreted as the reversible work required to extend a 
surface or to bring atoms from the interior to the surface region.

There is now wealth of spectroscopic and other analytical techniques for probing the surface properties of solid materials Brady [10] which yield a variety of surface properties of those parts of such materials that are situated anywhere between 1.0 and more than $10 \mathrm{~nm}$ below their surfaces [11]. Contact angle technique has been reported as being capable of yielding the actual surface or interfacial properties at the precise surfaces of solids that are relevant to their interaction with other condensed phase materials [12].

However, in this work, the energy in the interacting systems will be determined from the concept of Hamaker coefficients determined from Lifshitz theory Lifshiftz, et al. [13] which is based on spectrophotometric data.

\section{Methodology}

\subsection{Major Considerations}

The approach in this study is to collect some commonly used antiretroviral drugs, dissolve each in water and produce a film of the drug on a glass slide. In addition, the drugs will also be added to blood and thin films formed on glass slides. The popular technique can then be employed to determine the contact angles with given liquids, or alternatively, adopt a technique that has recently been used to determine the energies of interaction from spectrophotometric data. The latter is adopted in this work based on some confidence that has been reported in their use.

\subsection{Sample Collection}

The popular and commonly used unexpired antiretroviral drugs (three single tablets and two HAART), from the University of Nigeria Teaching Hospital (UNTH) APIN CENTRE PEPFAR, Ituku-Ozalla, Enugu State were collected. Table 1 shows the details of the five different antiretroviral drugs used in the study. Drugs 1 and 2 are both Highly Active Antiretroviral Therapy (HAART) as well as Fixed Dose Combination (FDC), while drugs 3, 4 and 5 are single antiretroviral drugs. Drugs 1,3 and 5 are administered to HIV patients twice daily while drugs 2 and 4 are taken once a day. It is worthy to note that all the antiretroviral drugs used were not expired during the period of the experiments.

Some quantities of blood samples were obtained through laboratory technologists at the Nnamdi Azikiwe University Teaching Hospital (NAUTH) Nnewi and Anambra State Teaching Hospital, Amaku (this was made possible by the Ethical Clearance, ANSUTH/AA/VOL.XI/008 of August 13, 2015, issued by Anambra State University Teaching Hospital, Amaku, Awka), as follows:

- from ten HIV infected persons who had not commenced drugs treatment (No ARV),

- from ten HIV infected persons that had already commenced treatment with antiretroviral drugs (With ARV), and

- from ten HIV negative persons.

Altogether, a total of thirty samples from different individuals were collected and screened to determine the infection status and stored in anticoagulant test tubes and ice packs to ensure the freshness and to avoid the samples becoming lysed. The samples were thereafter stored in a refrigerator for proper preservation [14].

\subsection{Lymphocytes Cell Count}

The CD4 counts of blood samples collected from ten HIV infected patients that had commenced ARV treatment (With ARV) and ten HIV patients that had not commenced treatment (No ARV) were initially determined using the CD4 counter (Cytoflowmeter) to ascertain the degree of infection of each blood sample with HIV. The results are given in Figure 1. The CD4 counts of the HIV positive patients who had not commenced treatment (No ARV) are lower than those for HIV positive patients who had commenced treatment (With ARV). Normally, the blood samples of the uninfected patients have higher CD4 counts above 500 cells $/ \mathrm{mm}^{3}$. It is noted that details of length of exposure of each patient to HIV or the time when treatment commenced, were not available. We can only say that these drugs have contributed to increased CD4 counts in the patients that took them.

\subsection{Sample Preparation}

The drugs passed through serial dilution at Tahilah Diagnostic Laboratories, Awka, in order to get the right con- 
Table 1. The details of the five different antiretroviral drugs used in the study [15].

\begin{tabular}{|c|c|c|c|c|c|c|}
\hline $\begin{array}{c}\text { Drug } \\
\text { Number }\end{array}$ & Tablets & $\begin{array}{l}\text { Abbrevi } \\
\text { Ation }\end{array}$ & Size & Batch Number & Expiration Date & Pharmaceutical Company \\
\hline 1 & $\begin{array}{c}\text { Lamivudine, Nevirapine \& } \\
\text { Zidovudine }\end{array}$ & $\begin{array}{c}3 \mathrm{TC}+\mathrm{NVP}+ \\
\text { ZDV }\end{array}$ & $\begin{array}{l}150 \mathrm{mg} / \\
200 \mathrm{mg} / \\
300 \mathrm{mg}\end{array}$ & 7220929 & $01 / 2016$ & Strides Arcolab Limited \\
\hline 2 & $\begin{array}{c}\text { Tenofovir, Lamivudine \& } \\
\text { Efavirenz }\end{array}$ & $\begin{array}{c}\mathrm{TDF}+3 \mathrm{TC}+ \\
\mathrm{EFV}\end{array}$ & $\begin{array}{l}300 \mathrm{mg} / \\
300 \mathrm{mg} / \\
600 \mathrm{mg}\end{array}$ & 3018522 & 09/2015 & Mylan Laboratories Limited \\
\hline 3 & Nevirapine & NVP & $200 \mathrm{mg}$ & 7216348 & $04 / 2015$ & Strides Arcolab Limited \\
\hline 4 & Efavirenz & EFV & $600 \mathrm{mg}$ & E121035A & $07 / 2015$ & HETERO LABS LIMITED \\
\hline 5 & Lamivudine & 3TC & $150 \mathrm{mg}$ & LEX -023 & $04 / 2016$ & $\begin{array}{l}\text { MCNEIL \& DRUGS } \\
\text { Pharmaceuticals Ltd. }\end{array}$ \\
\hline
\end{tabular}

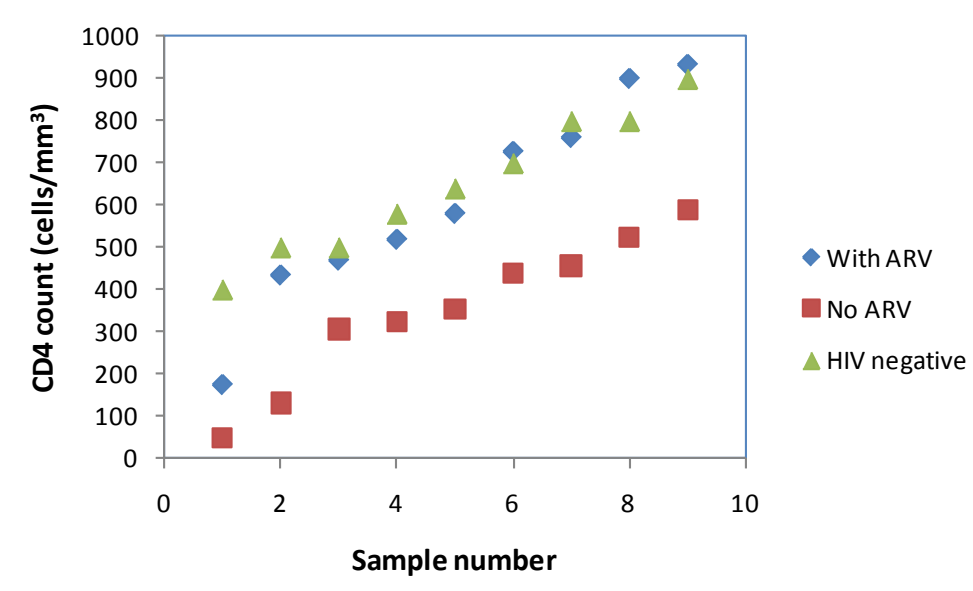

Figure 1. The CD4 count for blood samples.

centration of drug in the blood. After the serial dilutions to $10^{-2}$, the drug solution mixed with the blood was incubated at normal body temperature $\left(37^{\circ} \mathrm{C}\right)$ to facilitate drug-blood interactions. The knowledge of the onset and duration of action of each drug was used in administering the start dose and the maintenance dose in the blood samples. These collected samples with drug concentrations were loaded into a centrifugal separator and the blood components were separated at Tahilah Diagnostic Laboratories, Awka. This helped to obtain such components as White Blood Cells (WBC) also called the Lymphocytes, Red Blood Cells (RBC), and the Plasma or Serum, each sample at a time. Glass slides were prepared and smeared with the samples for absorbance measurements. The slide preparations and sample smearing were done at the same laboratory. About 600 slides were successfully prepared at 20 slides per sample.

\subsection{Measurements}

To effect the thermodynamic predictions, values of the absorbance data required for quantification of relevant information on the interacting systems, must normally be known. Absorbance measurements were done on all the different components of thirty samples (HIV infected blood of ones that had not started ARV treatment, HIV infected blood of those that had started ARV treatment and uninfected blood samples). A digital Ultraviolet Visible MetaSpecAE1405031Pro Spectrophotometer was used at the laboratory of the Department of Mechanical Engineering, Nnamdi Azikiwe University, Awka for the measurements. The absorbance values of the samples were measured over a range of wavelength between 230 and $970 \AA$ alongside with their corresponding transmittance values. The data collected Ani [15] were used to obtain the graphs presented in this research work.

\subsection{Concept of Hamaker Coefficients}

The thermodynamic model used for HIV-blood interactions is based on the concept of Hamaker coefficients as 
advanced by some authors [13] [16]-[20]. The idea is that there will be mutual attraction between the virus and the lymphocytes if the combined Hamaker coefficient $A_{132}$, is positive, and repulsion if it is negative.

To explain the concept of Hamaker Constants, van der Waals explanation of the derivations of the ideal gas law is used thus:

$$
P V=R T
$$

It was discovered that the kinetic energy of the molecules which strike a container wall is less than that of the bulk molecules. This effect was explained by the fact that the surface molecules are attracted by the bulk molecules even when the molecules have no permanent dipoles. It then follows that molecules can attract each other by some kind of cohesive force [21]. These forces have come to be known as van der Waals forces. van der Waals introduced the following corrections to Equation (1):

$$
\left[P+\frac{a}{V^{2}}\right](V-b)=R T
$$

The correction term to the pressure, $\left(\frac{a}{V^{2}}\right)$ indicates that the kinetic energy of the molecules which strike the container wall is less than that of the bulk molecules. This signifies the earlier mentioned attraction between the surface molecules and the bulk molecules.

After the development of the theory of quantum mechanics, London quantified the van der Waals statement for molecules without a dipole and so molecular attraction forces began to be known as London/van der Waals forces [22].

Hamaker made an essential step in 1937 from the mutual attraction of two molecules. He deduced that assemblies of molecules as in a solid body must attract other assemblies. The interaction energy can be obtained by the summation of all the interaction energies of all molecules present.

The analysis results in a van der Waals pressure, $P_{v d w}$ of attraction between two semi infinite (solid) bodies at a separation distance, $d$ in vacuum;

$$
P_{v d w}=\left[\frac{A_{11}}{6 \pi d^{3}}\right]
$$

where $A_{11}$ is the Hamaker Constant. This is the non-geometrical contribution to the force of attraction, based on molecular properties only.

For combination of two different materials 1 and 2 in approximation:

$$
A_{12}=\sqrt{A_{11} A_{22}}
$$

For a combination of three materials when the gap between 1 and 2 is filled with a medium 3 , then the following relations can be used:

$$
\begin{aligned}
& A_{131}=A_{11}+A_{33}-2 A_{13} \\
& A_{232}=A_{22}+A_{33}-2 A_{23}
\end{aligned}
$$

and;

$$
A_{132}=A_{12}+A_{33}-A_{13}-A_{23}
$$

Rewriting these equations will give.

And:

$$
A_{132}=\left(\sqrt{A_{11}}-\sqrt{A_{33}}\right)\left(\sqrt{A_{12}}-\sqrt{A_{33}}\right)
$$

Equation (8) shows that, for a three-component system involving three different materials, 1, 2 and 3, the combined Hamaker coefficient $A_{132}$ can become negative:

$$
A_{132} \prec 0
$$

When,

$$
A_{11}<A_{33}<A_{22} \text { or } A_{11}>A_{33}>A_{22}
$$


The implication of this is that two adhering bodies 1 and 2 of different composition will separate spontaneously upon immersion in a liquid 3 provided the conditions given by Equation (10) are fulfilled.

The limitations of Hamaker's approach led Lifshitz et al. [13] to develop an alternative derivation of van der Waals forces between solid bodies. The interaction between solids on the basis of their macroscopic properties considers the screening and other effects in their calculations. The equation was further approximated by Ninham and Parsegian [23], Isrealachvili [18], Nir, et al. [16], and Visser [17] because it is rather difficult to use. The "physical" meaning of the approximate equation was demonstrated by Krupp [24]. He showed that for a group of materials like polymers, the curves generated are identical while starting at a different position at zero frequency. Applying the absorption data of polystyrene, the value of $A_{11}$ becomes:

$$
A_{i i}=2.5\left[\frac{n_{i}^{2}-1}{n_{i}^{2}+1}\right]^{2}
$$

where $n_{i}$ is the refractive index of the polymer at zero frequency, it being bulk material property which can easily be obtained. Equation (11) has been used successfully in different applications and so we have confidence in its applicability in the systems under consideration.

To be able to use Equation (11), the refractive index must be determined from absorbance data through use of appropriate expressions relating refractive index to transmittance, reflectance and the absorbance.

\section{Results and Discussion}

\subsection{Infected Blood of Persons That Had Already Commenced Treatment with Antiretroviral Drugs (with ARV)}

The absorbance values measured for each of the ten samples were averaged; it was the average values for each blood component incubated in each antiretroviral drug for those that had previously started drug treatment that were plotted as a function of the wavelength, as given in Figure 2.

\subsection{HIV Infected Blood of Persons Who Were Not Yet on HIV Drugs (No ARV)}

The absorbance values measured for each of the ten samples were averaged; it was the average value for each blood component incubated in each antiretroviral drug for those that had not previously started drug treatment that was plotted as a function of the wavelength, are given in the preceding Figure 3.

Figure 2 and Figure 3 give the absorbance data plotted as a function of wavelength for the HIV infected blood components treated with each drug. The following features are observed:

- Each blood sample, whether infected with HIV or not, exhibits a maximum in its absorbance.

- The graph for each blood sample has a different peak absorbance value.

- The whole blood and red blood cells samples exhibit maximum absorbance at a wavelength of $410 \AA$ while the white blood cells and plasma samples exhibit maxima in absorbance at a wavelength of $320 \AA$.

- The wavelengths at which the maxima occurred are not affected by drug treatment of the blood component.

- On the whole, the peak absorbance values for the five antiretroviral drugs on lymphocytes range from 0.08 to 0.18 , on Plasma they range from 0.08 to 0.20 , on Red blood cells they range from 0.80 to 1.58 , for those that had commenced treatment with antiretroviral drugs, see Table 2.

- The peak absorbance values for the five antiretroviral drugs on Lymphocytes range from 0.18 to 0.30 , on the Plasma they range from 0.08 to 0.28 , on Red blood cells they range from 0.60 to 2.10 , for those that had not commenced treatment with antiretroviral drugs, see Table 2. All these fall within the visible range of the ultraviolet radiation which is $300-600 \AA$.

\subsection{HIV Negative Persons}

The absorbance values measured for each of the ten samples of HIV negative patients were averaged; it was the average value for each blood component incubated in each antiretroviral drug for HIV negative patients that was plotted as a function of the wavelength by Ani [15]. The peak absorbance values from this work are also listed in Table 2. 


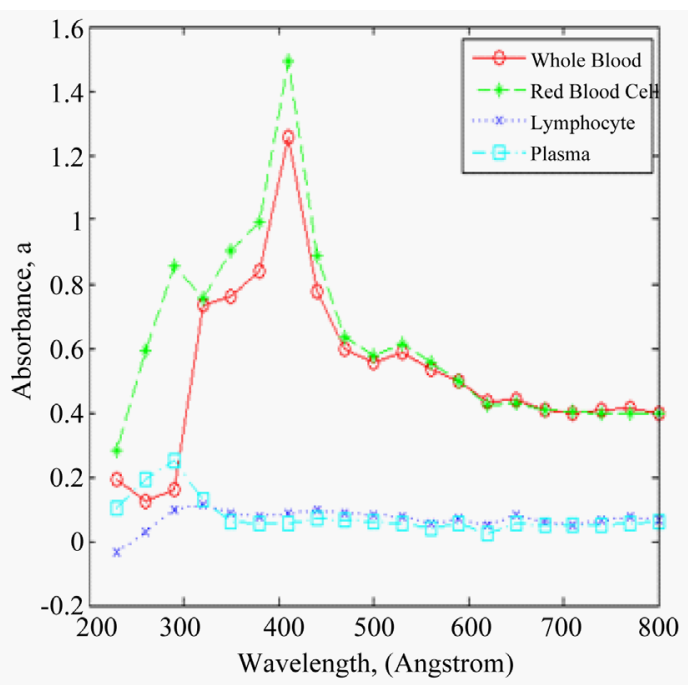

(a)

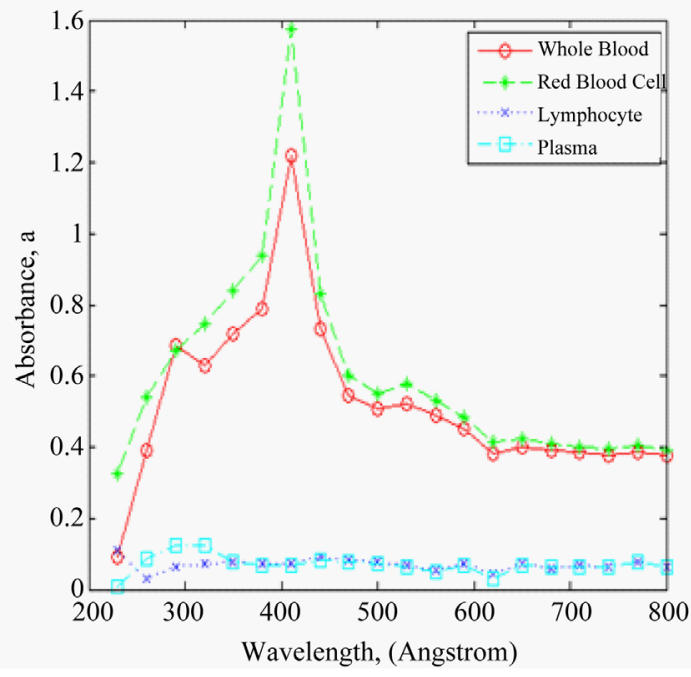

(c)

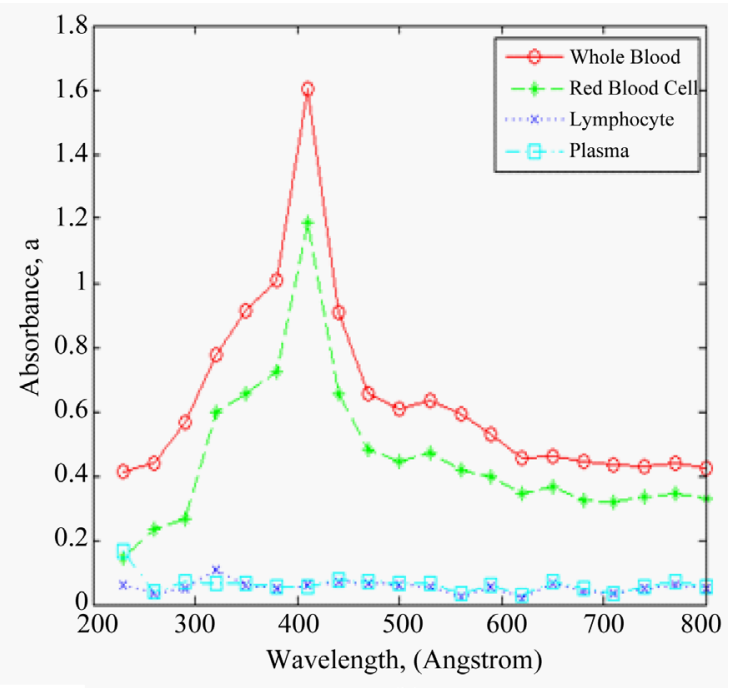

(b)

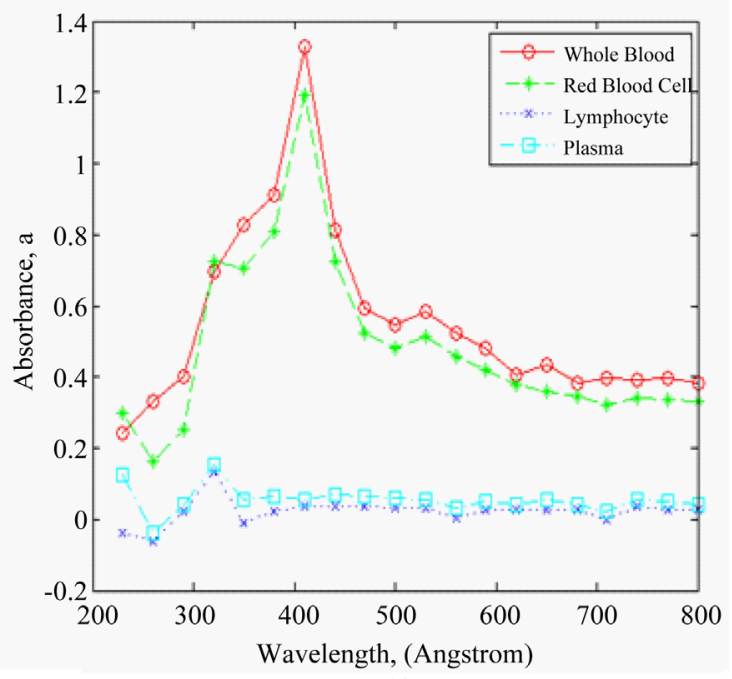

(d)

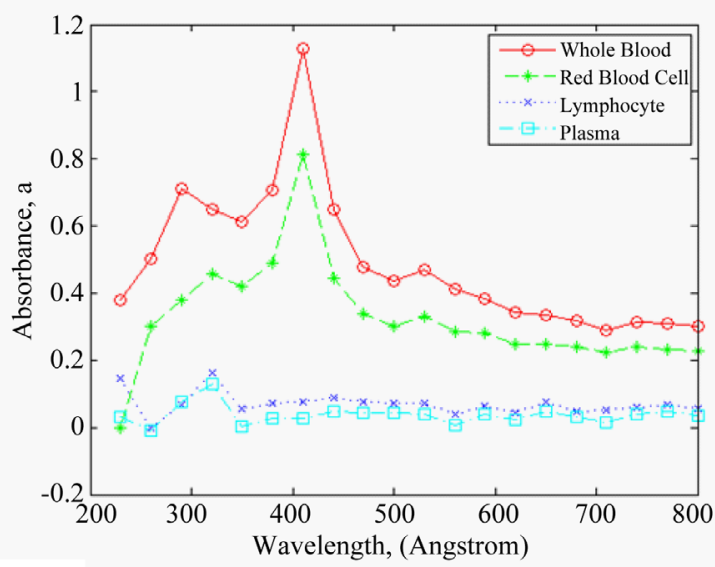

(e)

Figure 2. Absorbance, á versus Wavelength, $\lambda$ for HIV positive blood of patients that had commenced antiretroviral drug treatment before this study. (a) Drug 1: 3TC + NVP + ZDV; (b) Drug 2: TDF + 3TC + EFV; (c) Drug 3: NVP; (d) Drug 4: EFV; (e) Drug 5: 3TC. 


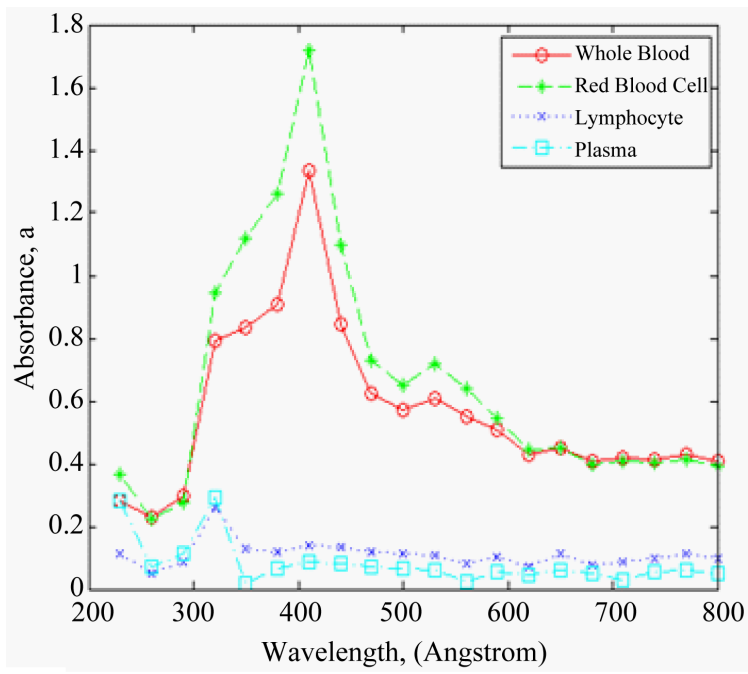

(a)

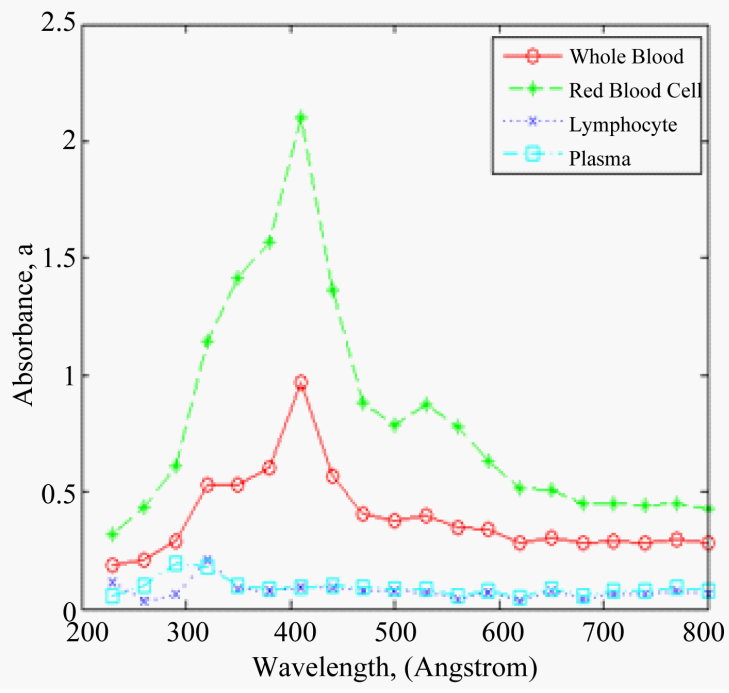

(c)

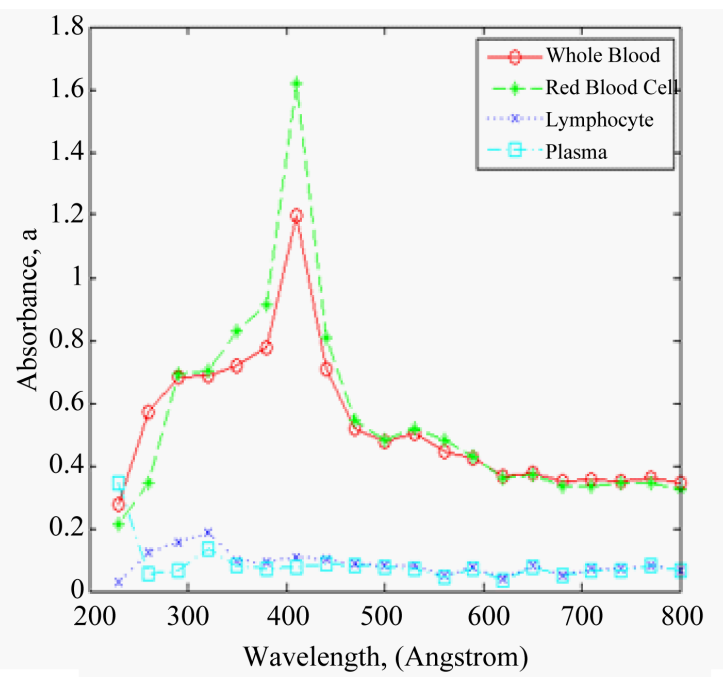

(b)

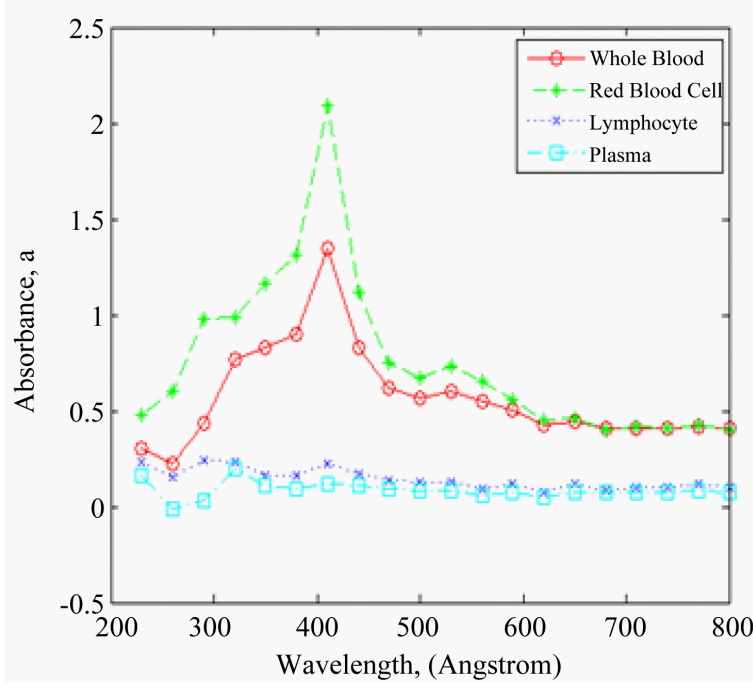

(d)

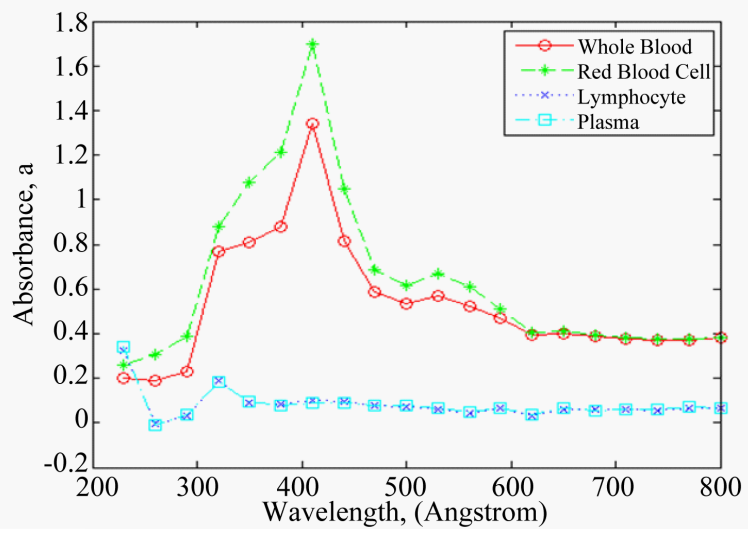

(e)

Figure 3. Absorbance, á versus Wavelength, $\lambda$ for for HIV positive blood components of patients that had not previously commenced antiretroviral treatment. (a) Drug 1: 3TC + NVP + ZDV; (b) Drug 2: TDF + 3TC + EFV; (c) Drug 3: NVP; (d) Drug 4: EFV; (e) Drug 5: 3TC. 


\subsection{Effects of the Drugs on the Absorbance}

The question that arises in the analysis of the data of Table 2 is: What is the combined effect of the drug and HIV on the surface of the blood component? This question has somehow been answered but additional work is presented through a mathematical model proposed to estimate these effects.

This model considers the effect of the antiretroviral drug on the surface of a blood cell, the effect of HIV and the combined effect of both the drug and the HIV, the final expression given by Ani, et al. [14] is:

$$
\eta_{d h}=\left(\frac{\tilde{\alpha}_{d}-\tilde{\alpha}_{o}}{\tilde{\alpha}_{d}}\right)\left(\frac{\tilde{\alpha}_{d}-\tilde{\alpha}_{h}}{\tilde{\alpha}_{d}}\right)
$$

where $\tilde{\alpha}_{d}$ is peak absorbance for drug film on blood component, and $\tilde{\alpha}_{o}$ is peak absorbance for blood component alone. The first term on the right hand side of the equation, which is the drug effect, is actually saying that, from absorbance concept, the difference the drug film makes in the absorbance of a blood component when compared with that of the absorbance of the blood component alone is some measure of drug effect. The antiretroviral drug has the capacity to increase the absorbance of a given blood component surface.

HIV has the effect of reducing the absorbance of the surface of a given blood component. The amount by which the surface of a blood component surface is decreased by HIV will be given by the second term on the right hand side. If HIV fails to reduce the absorbance of a drug film coated blood surface, it means that the HIV has not attached itself to the surface, then $\tilde{\alpha}_{h}=\tilde{\alpha}_{d}$ and the effect of HIV will be zero.

The above analysis will give us the idea of the effect of both the HIV and the antiretroviral drugs acting together on the blood and its components.

Using the relevant data of Table 2 together with the above proposed expression, the combined effect was calculated and listed in Table 3.

Table 3 shows the combined effect of coating of the HIV infected blood components with the antiretroviral drugs. The effectiveness of coating of the antiretroviral drugs on red blood cells gave varied, low and inconsistent results. Here, they are so small that it is clear that the antiretroviral drugs do not have any discernible and reproducible effect on the red blood cells. The covariance, which is the average of the products of deviations

Table 2. Data for peak absorbance for blood components of patients: $\alpha_{+\mathrm{m}}=$ HIV + ve with medication $\left(\mathrm{With}\right.$ ARV), $\alpha_{+\mathrm{n}}=$ HIV + ve without medication (No ARV), $\alpha_{0_{0}}=$ HIV negative.

\begin{tabular}{cccccccccccc}
\hline \multirow{3}{*}{ Drug No. } & \multicolumn{3}{c}{ RBC } & \multicolumn{3}{c}{ WBC } & \multicolumn{3}{c}{ Plasma } \\
${$\cline { 2 - 6 }$y} y }$ & $\alpha_{+\mathrm{m}}$ & $\alpha_{+\mathrm{n}}$ & $\alpha_{-\mathrm{o}}$ & $\alpha_{+\mathrm{m}}$ & $\alpha_{+\mathrm{n}}$ & $\alpha_{-\mathrm{o}}$ & $\alpha_{+\mathrm{m}}$ & $\alpha_{+\mathrm{n}}$ & $\alpha_{-\mathrm{o}}$ \\
\hline $\mathbf{1}$ & 1.50 & 1.60 & 1.50 & 0.14 & 0.18 & 0.48 & 0.20 & 0.16 & 0.22 \\
$\mathbf{2}$ & 1.20 & 1.76 & 1.88 & 0.10 & 0.26 & 0.52 & 0.08 & 0.08 & 0.18 \\
$\mathbf{3}$ & 1.58 & 2.10 & 1.90 & 0.08 & 0.20 & 0.30 & 0.12 & 0.19 & 0.26 \\
$\mathbf{4}$ & 1.20 & 2.08 & 2.28 & 0.17 & 0.30 & 0.28 & 0.18 & 0.28 & 0.30 \\
$\mathbf{5}$ & 0.80 & 1.70 & 2.18 & 0.18 & 0.19 & 0.27 & 0.16 & 0.20 & 0.20 \\
\hline
\end{tabular}

Table 3. Combined effect of coating, $\eta_{d h}$.

\begin{tabular}{cccccc}
\hline & \multicolumn{2}{c}{ HIV infected without previous drug treatment } & \multicolumn{3}{c}{ HIV infected with previous drug treatment } \\
\cline { 2 - 5 } Drug & WBC & RBC & Plasma & WBC & 0.0000 \\
\hline $\mathbf{1}$ & 0.5102 & 0.0108 & 0.1809 & 0.5782 & 0.0535 \\
$\mathbf{2}$ & 0.4152 & 0.0005 & 0.3269 & 0.6707 & 0.0262 \\
$\mathbf{3}$ & 0.2353 & -0.0009 & 0.1925 & 0.5177 & 0.0138 \\
$\mathbf{4}$ & 0.0245 & 0.0206 & 0.0502 & 0.2691 & 0.1114 \\
$\mathbf{5}$ & 0.1995 & 0.0440 & 0.1632 & 0.2244 & 0.3012 \\
\hline
\end{tabular}


between the data of WBC and RBC is -0.0012 and for WBC and plasma, it is 0.011 for blood that had not received prior treatment before this test. However, for the blood that had received previous drug treatment, the variance between WBC and RBC is -0.008 and between WBC and plasma, it is -0.002 . Pearson rho also gave a negative correlation $(-0.42)$ coefficient. These low and/or negative variations and correlations show that the absorbance data for WBC do not correlate with those of RBC and plasma, confirming the fact that the antiretroviral drugs are specifically designed to target white blood cells. Further considerations of HIV-blood interactions in antiretroviral environment will therefore dwell on HIV-WBC interactions.

\subsection{HIV-Blood Interactions}

The thermodynamic models for HIV-blood interactions have been presented above. The challenge lies with being able to determine the values of the Hamaker coefficient. It has been variously shown that, by employing the classical Lifshitz theory in conjunction with the absorbance data, the Hamaker constants and hence the Hamaker coefficients can be calculated. This equation has been successfully applied in biological systems interactions [16] [24]. For this study, the symbols for the various Hamaker constants will be as follows:

$$
\begin{aligned}
& A_{33}=\text { Hamaker constant for plasma-drug treated HIV positive or negative serum; } \\
& A_{11}=\text { Hamaker constant for lymphocyte-drug coated HIV negative lymphocytes; } \\
& A_{22}=\text { Hamaker constant for HIV-drug coated HIV positive lymphocytes. }
\end{aligned}
$$

The infected lymphocytes are used in lieu of the virus because there is currently no known means of isolating the virus. The assumption here is that the infected lymphocyte is an approximation of the actual virus owing to the manner of the infection. The mechanism of the viral infection is such that it actually attaches its CD8+ cells on the wild CCR5 dendrites of the blood CD4+ T4 cells and thereby changing the nature of the cells. Thus use is made of the infected lymphocytes as a close approximation for the virus in calculating the Hamaker constants.

The MATLAB software tools were employed in the mathematical analysis of the very large body of data generated from the experiments. The Hamaker constants $A_{11}, A_{22}, A_{33}$ and the combined Hamaker coefficients $A_{132}, A_{232}$ and $A_{131}$ of the various drugs interacting with the blood were obtained using the values of the dielectric constant together with the approximate Lifshiftz equation Equation (7). For each sample, there are twenty slides, thus the absolute value of the Hamaker constant is the average over all the data for the twenty slides. The Hamaker constants and Hamaker coefficients calculated using the absorbance data are listed in Table 4 for WBC.

Table 4 reveals that $A_{11}$ is greater than $A_{33}$ (in all cases) which in turn is greater than $A_{22}$ for each antiretroviral drug in HIV positive blood without antiretroviral treatment (NARV). This is in agreement with the condition for rendering the combined Hamaker coefficient, $A_{132}$ negative (Equations (9) and (10)). This indicates that, from our thermodynamic model, the five different antiretroviral drugs used in the study can to some degree and for blood without previous drug treatment (NARV), render the Hamaker coefficients negative as also given in Table 4. This means that the van der Waals forces are repulsive indicating the possibility of the drug-coated

\begin{tabular}{|c|c|c|c|c|c|c|c|c|}
\hline \multirow{3}{*}{ Drug No. } & \multicolumn{5}{|c|}{ Hamaker constants } & \multicolumn{3}{|c|}{ Hamaker coefficients } \\
\hline & $\begin{array}{c}A_{11} \\
\left(10^{-21} \text { Joule }\right)\end{array}$ & $\underset{\left(10^{-21} \text { Joule }\right)}{A_{22}}$ & $\underset{\left(10^{-21} \text { Joule }\right)}{A_{22}}$ & $\begin{array}{c}A_{33} \\
\left(10^{-21} \text { Joule }\right)\end{array}$ & $\begin{array}{c}A_{33} \\
\left(10^{-21} \text { Joule }\right)\end{array}$ & $\begin{array}{c}A_{131} \\
\left(10^{-21} \text { Joule }\right)\end{array}$ & $A_{132}\left(10^{-2}\right.$ & Joule) \\
\hline & HIV -ve & $\begin{array}{l}\text { HIV +ve } \\
\text { (ARV) }\end{array}$ & $\begin{array}{l}\text { HIV +ve } \\
\text { (NARV) }\end{array}$ & $\begin{array}{l}\text { HIV +ve } \\
\text { (ARV) }\end{array}$ & $\begin{array}{l}\text { HIV +ve } \\
\text { (NARV) }\end{array}$ & HIV -ve & HIV +ve (ARV) & $\begin{array}{l}\text { HIV +ve } \\
\text { (NARV) }\end{array}$ \\
\hline 1 & 1.1869 & 0.2933 & 0.2768 & 1.8190 & 0.4588 & 0.3676 & 0.4095 & -0.0400 \\
\hline 2 & 1.0522 & 0.2544 & 0.3475 & 1.0818 & 0.5711 & 0.4634 & 0.1027 & -0.0531 \\
\hline 3 & 1.0836 & 0.3344 & 0.2323 & 0.9580 & 0.4008 & 0.5302 & -0.2505 & -0.0585 \\
\hline 4 & 0.9866 & 0.3320 & 0.3782 & 1.0920 & 0.4913 & 0.5097 & 0.0716 & -0.0248 \\
\hline 5 & 1.1200 & 0.2833 & 0.2925 & 1.1012 & 0.4819 & 0.4960 & -0.1040 & -0.0584 \\
\hline
\end{tabular}
lymphocytes repelling or blocking the HIV from attacking the lymphocytes.

Table 4. Comparison of Hamaker constants and Hamaker coefficients. 
For clarity, values of $A_{132}$ in Table 4 are presented in a bar chart and shown in Figure 4 that clarifies the positive and negative senses of the values. The absolute combined Hamaker coefficients for drugs 3 and 5 of the HIV positive blood with antiretroviral treatment (ARV) have negative values while the interacting systems involving drugs 1,2 and 4 gave positive values. The values for $A_{132}$ are all negative for all the five different antiretroviral drugs in HIV positive lymphocytes without antiretroviral treatment (NARV). These negative values of the absolute combined Hamaker coefficients indicate a van der Waals repulsion in the HIV positive systems initiated by the administration of the antiretroviral drugs. This results in increase of the interaction energy of the infected blood. Thus, the possibilty of drug coated lymphocyte blocking the attachment of HIV to its surface is hightened. If the virus does not adhere to the surface of the lymphocyte, then with time the virus in the blood will die off. This is particularly so since the viral replication takes place only when HIV fuses with the host cell and attacks the DNA after which it translates to RNA making more HIV copies which are released after maturation. Thus, the entire HIV in the infected patient may "die" off, enhancing possible elimination of the virus in the blood stream and thus making room for increased CD4 cells count. This conclusion is in agreement with the speculations of Achebe [20].

Moreover, the positive values of the absolute combined Hamaker coefficients indicate a van der Waals attraction between the lymphocytes and the antiretroviral drugs in the HIV negative systems.

It appears that prior treatment with antiretroviral drugs has not produced much advantage in this case which is contrary considering the conclusion from the coating effectiveness.

\subsection{Deductions for the Absolute Combined Negative Hamaker Coefficients of the Interacting Systems}

The absolute combined Hamaker coefficient, $A_{132 a b s}$ and $A_{131 a b s}$ (an integral of all the values of the various Hamaker coefficients) for each drug as well as for all drugs on both infected and uninfected blood samples were calculated and listed in Table 5. The underlying equations here are given by Equation (13) [15] [19]:

$$
A_{132 \mathrm{abs}}=\frac{\sum_{0}^{N}\left(A_{132}\right)}{N} ; A_{131 \mathrm{abs}}=\frac{\sum_{0}^{N}\left(A_{131}\right)}{N} ; A_{232 \mathrm{abs}}=\frac{\sum_{0}^{N}\left(A_{232}\right)}{N}
$$

Table 5 reveals that the interaction energy among the lymphocytes, $A_{131}$ is greater than the interaction energy among the virus (HIV) infected lymphocytes, $A_{232}$, the difference varying from about $42 \%$ with drug 1 to about

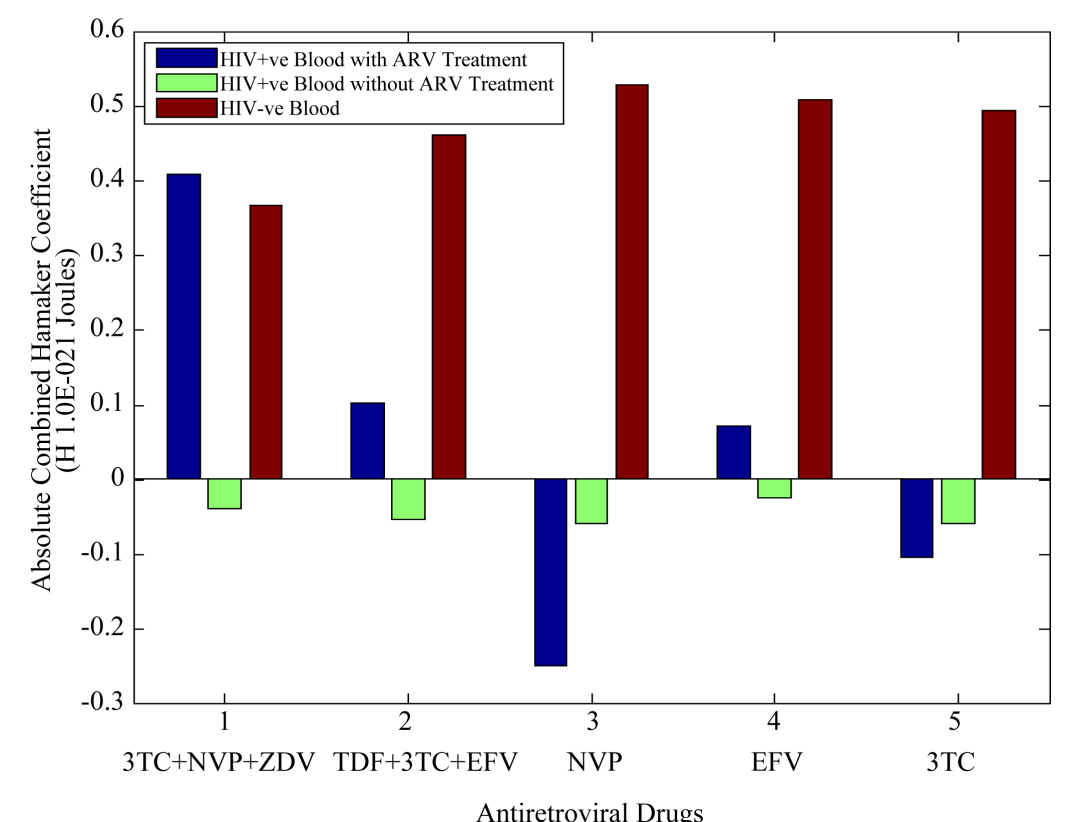

Figure 4. Plot of absolute combined Hamaker coefficient of each antiretroviral drug for different interacting systems versus the antiretroviral drugs used. 
Table 5. Comparison between the absolute values of $A_{131}$ for uninfected lymphocytes and $A_{132}, A_{232}$ for infected lymphocytes for the five different antiretroviral drugs.

\begin{tabular}{cccccc}
\hline Variable $\left(\times \mathbf{1 0}^{-\mathbf{2 1}}\right.$ Joule $)$ & D1 & D2 & D3 & D4 & D5 \\
\hline $\boldsymbol{A}_{\mathbf{1 3 1}}$ & 0.367603 & 0.463371 & 0.530208 & 0.509707 & 0.495986 \\
$\boldsymbol{A}_{\mathbf{2 3 2}}$ & 0.213619 & 0.276577 & 0.141540 & 0.186744 & 0.144346 \\
$\boldsymbol{A}_{\mathbf{1 3 2}}$ & -0.03998 & -0.05305 & -0.05845 & -0.02481 & -0.05844 \\
\hline
\end{tabular}

73\% with drug 3 . This shows that the virus actually has the tendency to weaken the lymphocyte, as expected. The positive values of the absolute combined Hamaker coefficient $A_{131}$ for each antiretroviral drug interacting with HIV negative WBC indicate that there is an attraction between the bodies. This is so because the positive sense of the absolute combined Hamaker coefficient shows that the van der Waals forces of the interacting system are attractive. In effect, this suggests that the lymphocytes are attracted to the antiretroviral drugs, which facilitates the binding or surface coating of the drug films on the lymphocytes surfaces.

The results of this study show that the combined Hamaker coefficients, $A_{132}$, which had been found to be positive for HIV infected lymphocytes (and hence strong attraction between them) Achebe [19] are rendered negative to different degrees by the addition of antiretroviral drugs (this suggests that there is force of repulsion between virus and blood component). This repulsion, in principle, suggests that the virus would not come in contact with the lymphocyte as to adhere on it and attack it. All the drugs studied showed that propensity to varying degrees. One expects that the drug that gives the highest negative Hamaker coefficient will have the capacity to cause stronger repulsion between the virus and the lymphocyte. All these confirm the applicability of surface thermodynamics modeled using the concept of negative Hamaker coefficients, in the study of HIV-blood interactions in the presence of antiretroviral drugs.

Summary of the results of HIV-drug coated blood interactions is shown in Table 6. The various antiretroviral drugs are shown together with the absolute combined Hamaker coefficients $A_{132 \text { abs }}$ of each of the drugs in HIV positive or negative lymphocytes. The consequences of the interaction energies and the van der Waals forces, i.e., whether they are repulsive or attractive are also indicated.

Table 6 thus gives a bird's eye view on the consequences of interactions between HIV and the lymphocytes in the antiretroviral drugs environment.

Criteria for the prediction of possible elimination of HIV from blood stream are presented in Table 7. Previously, the efficacy of the antiretroviral drugs was checked virologically, immunologically and clinically using various markers such as viral loads, CD4 cells count and clinical stage respectively. Table 7 provides information on these markers as communicated by some researchers in the field like Moore AL et al. [25]. The suggested viral loads with periods of sustained treatment are indicated. It is expected that the viral load would have (by about a factor of eight) after 48 weeks of treatment been reduced to somehow very low level. Within this period the CD4 count would be estimated to increase by a factor of seven. Clinically, the HIV symptoms would be expected to have become probably unnoticeable.

From this work, the value of the absolute combined Hamaker coefficient in the HIV-drug coated lymphocytes interactions may be used as marker. Thus, the thermodynamic response on the steady administration of the antiretroviral drugs to HIV positive system is the change of the net repulsive van der Waals forces between HIV and drug-coated lymphocytes from positive to negative values, resulting to increase in surface energy of the HIV positive blood, maybe, within the first 48 weeks.

In summary, the virological response on the steady administration of the antiretroviral drugs to HIV positive blood is the reduced viral loads to clinically undetectable levels within the first 48 weeks, the immunological response on the steady administration of the antiretroviral drugs to HIV positive blood is the increased CD4 cells count to at least 350 cells $/ \mathrm{mm}^{3}$ within the first 48 weeks, the clinical response on the steady administration of the antiretroviral drugs to HIV positive system is that the symptoms of HIV infections seemingly abate or subside within the first 12 weeks of treatment initiation, and the surface thermodynamic approach suggests change in the interaction energy from positive to negative.

\section{Conclusions}

In the twenty first century research works, there is a growing need to achieve a more reliable research result 
Table 6. Summary of the results of HIV-drug coated lymphocytes interactions.

\begin{tabular}{|c|c|c|c|c|c|c|c|c|}
\hline Drug No. & Drug type & ARV & $\begin{array}{c}A_{132 a b s} \\
\left(\times 10^{-21} \text { Joule }\right) \\
\text { HIV Positive } \\
\text { Blood }\end{array}$ & $\begin{array}{l}\text { van der Waals } \\
\text { Forces }\end{array}$ & $\begin{array}{l}\text { Nature of } \\
\text { Interaction }\end{array}$ & $\begin{array}{l}A_{131 a b s}\left(\times 10^{-21}\right. \\
\text { Joule) of HIV } \\
\text { Negative Blood }\end{array}$ & $\begin{array}{c}\text { van der Waals } \\
\text { Forces }\end{array}$ & $\begin{array}{l}\text { Nature of } \\
\text { Interaction }\end{array}$ \\
\hline 1 & $\begin{array}{l}\text { HAART and } \\
\text { FDC }\end{array}$ & $\begin{array}{l}\text { Lamivudine, } \\
\text { Nevirapine \& } \\
\text { Zidovudine }\end{array}$ & -0.03998 & $\begin{array}{c}\text {-ve } \\
\text { Repulsive }\end{array}$ & Repulsion & 0.367603 & $\begin{array}{c}\text { +ve } \\
\text { Attractive }\end{array}$ & Attraction \\
\hline 2 & $\begin{array}{l}\text { HAART and } \\
\text { FDC }\end{array}$ & $\begin{array}{l}\text { Tenofovir, } \\
\text { Lamivudine \& } \\
\text { Efavirenz }\end{array}$ & -0.05305 & $\begin{array}{c}\text {-ve } \\
\text { Repulsive }\end{array}$ & Repulsion & 0.463371 & $\begin{array}{c}\text { +ve } \\
\text { Attractive }\end{array}$ & Attraction \\
\hline 3 & Single Drug & Nevirapine & -0.05845 & $\begin{array}{c}\text {-ve } \\
\text { Repulsive }\end{array}$ & Repulsion & 0.530208 & $\begin{array}{c}\text { +ve } \\
\text { Attractive }\end{array}$ & Attraction \\
\hline 4 & Single Drug & Efavirenz & -0.02481 & $\begin{array}{c}\text {-ve } \\
\text { Repulsive }\end{array}$ & Repulsion & 0.509707 & $\begin{array}{c}\text { +ve } \\
\text { Attractive }\end{array}$ & Attraction \\
\hline 5 & Single Drug & Lamivudine & -0.05844 & $\begin{array}{c}\text {-ve } \\
\text { Repulsive }\end{array}$ & Repulsion & 0.495986 & $\begin{array}{c}\text { +ve } \\
\text { Attractive }\end{array}$ & Attraction \\
\hline
\end{tabular}

Table 7. The proposed criteria in HIV-drug interactions.

\begin{tabular}{|c|c|c|c|c|c|c|}
\hline Efficacy & \multicolumn{2}{|c|}{ Virological } & Immunological & Clinical & \multicolumn{2}{|c|}{ Thermodynamics } \\
\hline Marker & \multicolumn{2}{|c|}{ Viral load } & \multirow{2}{*}{$\begin{array}{l}\text { CD4 cells count } \\
24 \text { - } 48 \text { weeks }\end{array}$} & \multirow{2}{*}{$\begin{array}{l}\text { Clinical stage } \\
\text { By } 12 \text { weeks }\end{array}$} & \multicolumn{2}{|c|}{$\begin{array}{l}\text { Absolute combined Hamaker } \\
\text { coefficients }\end{array}$} \\
\hline Time & 24 weeks & 48 weeks & & & 24 weeks & 48 weeks \\
\hline Suggested range & $\begin{array}{l}<400 \text { copies } / \mathrm{ml} \\
\text { of HIV }\end{array}$ & $\begin{array}{c}<50 \text { copies } / \mathrm{ml} \\
\text { of HIV }\end{array}$ & $\begin{array}{l}\text { Increase from } \\
\text { baseline by at } \\
\text { least } 50-100 \\
\text { cells } / \mathrm{mm}^{3}\end{array}$ & Stage 1 or 2 & $\begin{array}{l}\text { Negative } A_{132 a b s} \text { for } \\
\text { HIV + ve blood and } \\
\text { positive } A_{131 \text { abs }} \text { for } \\
\text { HIV -ve blood }\end{array}$ & $\begin{array}{l}\text { Increased negative } \\
\mathrm{A}_{132 \mathrm{abs}} \text { for HIV +ve } \\
\text { blood and } \\
\text { decreased positive } \\
\mathrm{A}_{131 \mathrm{abs}} \text { for HIV -ve } \\
\text { blood }\end{array}$ \\
\hline Response & $\begin{array}{r}\text { Reduced viral l } \\
\text { undetect }\end{array}$ & $\begin{array}{l}\text { ads to clinically } \\
\text { ble levels }\end{array}$ & $\begin{array}{c}\text { Increased CD4 cell } \\
\text { count to at least } 350 \\
\text { cells } / \mathrm{mm}^{3}\end{array}$ & $\begin{array}{l}\text { Symptoms of HIV } \\
\text { infection abate or } \\
\text { subside }\end{array}$ & \multicolumn{2}{|c|}{$\begin{array}{l}\text { Net repulsive van der Waals forces } \\
\text { between HIV and drug treated } \\
\text { lymphocytes, resulting in increase in } \\
\text { surface energy of HIV + ve blood }\end{array}$} \\
\hline
\end{tabular}

through a synergy between engineers and biological researchers. Surface thermodynamics through the concept of negative Hamaker coefficient was adopted in the study of the interactions between HIV and antiretroviral drug treated blood.

The methodology involved the collection of five antiretroviral drugs and blood from HIV patients that had started antiretroviral drug treatment, from patients that had not started the drug treatment and from HIV negative patients. The blood was screened and carefully treated with each of the drugs as appropriate and then prepared and subjected to absorbance measurements.

The interaction energies, in terms of the Hamaker coefficients were calculated using an approximate expression of the Lifshitz equation together with the absorbance data. The absolute values for the combined Hamaker coefficient, $A_{132}$ obtained for each of the five antiretroviral drugs interacting with infected blood without antiretroviral treatment range from $-0.02481 \times 10^{-21}$ Joule for drug 4 to $-0.05845 \times 10^{-21}$ Joule for drug 3 . The negative values of the absolute combined Hamaker coefficients obtained for the five antiretroviral drugs on HIV positive lymphocytes indicate that there are repulsive van der Waals forces which suggest that the interacting bodies (that is HIV and drug-coated lymphocytes) repel each other. It may thus be reasoned that the antiretroviral drugs on the surface of the lymphocytes can successfully block the HIV at different stages of its replication. Another significance of this negative value of the absolute combined Hamaker coefficient is that the use of each of the five antiretroviral drugs may succeed in reducing the viral loads, improving the CD4 cells count as well as increasing the surface energy of the HIV positive lymphocyte.

This will serve as one of the criteria for HIV treatment success - the thermodynamic efficacy of the antiretro- 
viral drugs with the response of the body to the drugs as shown in Table 7.

On the other hand, the absolute values for the combined Hamaker coefficient, $A_{132}$ obtained for each of the five antiretroviral drugs interacting with infected lymphocytes with previous antiretroviral treatment show that the interacting systems involving drugs 3 and 5 gave a negative absolute combined Hamaker coefficients $A_{132}$ indicating a repulsion between the HIV and the lymphocytes while the interacting systems involving drugs 1,2 and 4 gave a positive absolute combined Hamaker coefficients $A_{132}$ indicating more van der Waals attractions between the newly administered antiretroviral drugs and the already existing drug-coated lymphocytes in the HIV positive systems (i.e drug-drug interactions) than HIV-drug coated blood interactions.

Also, the absolute values for the combined Hamaker coefficients, $A_{131}$ obtained for each of the five antiretroviral drugs interacting with uninfected blood range from $0.36760 \times 10^{-21}$ Joule for drug 1 to $0.53021 \times 10^{-21}$ Joule for drug 3 . The positive values of the absolute combined Hamaker coefficients obtained for the five antiretroviral drugs interacting with HIV negative blood indicate attraction between the surface of the lymphocyte and the drug film causing improved binding. Hence, the van der Waals forces of the interacting process are attractive.

The use of the concept of combined Hamaker coefficients as marker, along with other markers, in the assessment of the stage of the infection in the treatment of HIV with antiretroviral drugs was proposed. Since the antiretroviral drugs used as additive(s) to the serum serving as the intervening medium rendered the absolute combined Hamaker coefficients negative, the possibility of formulating a drug that can completely block the virus from the lymphocytes is not a remote possibility. This is the strength of the surface thermodynamics approach to HIV-blood interactions in antiretroviral drug environment.

\section{References}

[1] UNAIDS (2013) Report on the Global on HIV/AIDS Treatment.

[2] UNAIDS (2006) Report on the Global AIDS Epidemic/UNAIDS: A UNAIDS $10^{\text {th }}$ Anniversary Special Edition. 1-2.

[3] Szekeres, G. (1999) Bulletin of Experimental Treatments for AIDS. San Francisco AIDS Foundation.

[4] Omenyi, S.N. (2005) The Concept of Negative Hamaker Coefficients. Nnamdi Azikiwe University, Awka, Inaugural Lecture Series No. 8.1, 23.

[5] Quashie, P.K. (2013) HIV Drug Resistance and the Advent of Integrase Inhibitors. Current Infectious Disease Reports, 15, 85-100. http://dx.doi.org/10.1007/s11908-012-0305-1

[6] United States Department of Health and Human Services (2004) A Guide to Primary Care for People with HIV/AIDS.

[7] Achebe, C.H. and Omenyi, S.N. (2013) The Effects of Human Immunodeficiency Virus (HIV) Infections on the Absorbance Characteristics of Different Blood Components. International Journal of Science Invention, 2, 53-61. www.ijesi.org

[8] Good, R.J. (1979) Contact Angles and Surface Free Energy of Solids. Surface and Colloid Science, Plenum Press. http://dx.doi.org/10.1007/978-1-4615-7969-4_1

[9] Lyklema, J. (1991) Fundamentals of Interface and Colloid Science. Vol. 3, Liquid-Fluid Interfaces, Academic Press.

[10] Brady, P.V. (1996) Physical and Chemistry of Mineral Surfaces. CRC Press.

[11] Etzler, F.M. (2001) Characterization of Surface Free Energies and Surface Chemistry of Solids. In: Mittal, K.L., Ed., Contact Angle, Wettability and Adhesion, VSP, Utrecht, 219-264.

[12] van Oss, C.J. and Giese, R.F. (2002) Colloid and Surface Properties of Clay and Related Minerals. Marcel Dekker, New York.

[13] Lifshiftz, E.M., Dzyaloshinskii, I.E. and Pitaevskii, L.P. (1961) The General Theory of van der Waals Forces. Advances in Physics, 10, 165-209. http://dx.doi.org/10.1080/00018736100101281

[14] Ani, O.I., Omenyi, S.N. and Achebe, C.H. (2015) Negative Hamaker Coefficients: Application to the Human Immunodeficiency Virus (HIV)-Blood Interactions in Antiretroviral Drug Media. International Journal of Engineering and Applied Sciences (IJEAS), 11, 4-9. www.eaas-journal.org

[15] Ani, O.I. (2015) Surface Energetics Study of the Interactions between HIV and Blood Cells Treated with Antiretroviral Drugs. PhD Dissertation, Nnamdi Azikiwe University, Awka.

[16] Nir, S., Rein, R. and Weiss, L. (1972) On the Applicability of Certain Approximations of the Lifshitz Theory to Thin Films. Journal of Theoretical Biology, 34, 135-153. http://dx.doi.org/10.1016/0022-5193(72)90060-4

[17] Visser, J. (1975) In: Matijevic, E., Ed., Surface/Colloid Science, Volume 8, Wiley-Interscience, New York, 3. 
[18] Israelachivili, J.N. (1972) Proceedings of the Royal Social Services A, 331, 39.

[19] Achebe, C.H. (2010) Human Immunodefficiency Virus (HIV)-Blood Interactions: Surface Thermodynamics Approach. PhD Dissertation, Nnamdi Azikiwe University, Awka.

[20] Ozoihu, E.M. (2014) Human Immunodefficiency Virus (HIV)-Blood Interactions: Contact Angle Approach. PhD Dissertation, Nnamdi Azikiwe University, Awka.

[21] Van der Waals, J.D. (1873) Thesis, Leiden.

[22] London, F. (1930) Zur Theorie und Systematik der Molekularkräfte. Zeitschrift für Physik, 63, 245-279. http://dx.doi.org/10.1007/BF01421741

[23] Ninham, B.W. and Parsegian, V.A. (1970) van der Waals Forces across Triple-Layer Films. Journal of Chemistry and Physics, 52, 4578-4587. http://dx.doi.org/10.1063/1.1673689

[24] Krupp, H. (1967) Particle Adhesion Theory and Experiment. Advances in Colloid Interface Science, 1, 111-239. http://dx.doi.org/10.1016/0001-8686(67)80004-6

[25] Moore, A.L., Youle, M., Lipman, M., et al. (2002) Raised Viral Load in Patients with Viral Suppression on Highly Active Antiretroviral Therapy: Transient Increase or Treatment Failure? AIDS, 16, 615-618. 